\title{
Handover Prediction Strategy for 3G-WLAN Overlay Networks
}

\author{
Hyeyeon Kwon, Mi-Jeong Yang, Ae-Soon Park \\ Mobile Telecommunication Research Division \\ Electronics and Telecommunications Research Institute \\ Daejeon, 305-707, Korea \\ hykwon@etri.re.kr,mjyang@etri.re.kr,aspark@etri.re.kr
}

\author{
S.Venkatesan \\ Department of Computer Science \\ University of Texas at Dallas, \\ Richardson, TX 75083 \\ venky@utdallas.edu
}

\begin{abstract}
In 3G-WLAN overlay network environments, a UE is apt to lose the WLAN signal without any notice by moving while it connects to WLAN because it can be apart from the WLAN coverage suddenly. In this case the on-going services are disrupted by unexpected link corruption. Hence, it needs to execute quickly the handover to $3 G$ network as soon as it detects the lost of WLAN signal. However, as the 3G access has many network signaling operations additionally including IP mobility operations for service continuity, it requests more enough processing time for the operations, which increases the handover latency causing the service interruption. Therefore it is important to predict the time when the UE will get out of the WLAN coverage. By the handover prediction, the UE can prepare the connection to $3 G$ network before breaking off the WLAN connection. This paper shows several handover prediction algorithms to reduce the handover latency by fast handover triggering. The correctness of the algorithms is compared by simulation.
\end{abstract}

Keywords-VHO, handover prediction, seamless mobility

\section{INTRODUCTION}

3GPP (3G Partnership Projects) has taken into account the $3 \mathrm{G}$ and IEEE 802.11 WLAN (Wireless Local Area Network) interworking architecture as an add-on to the existing 3GPP system specifications to be published with 3GPP Release 6 and 7 specifications [1,2]. The main objective of the 3GPP activity is to extend the 3GPP services and functionality to the WLAN access environment. The final goal of 3GPP-WLAN interworking is seamless mobility which minimizes the latency and data loss during the handover $[3,4,5,6]$.

The WLANs may interwork with home and/or visited $3 \mathrm{G}$ systems and also the interworked WLANs may nest in $3 \mathrm{G}$ network coverage and overlap each other in hot-spots, as well as UTRAN (UMTS Terrestrial RAN) in UMTS (Universal Mobile Telecommunication System) [1]. In this kind of 3GWLAN overlay network, as WLAN can be a preferred network that provides better throughput performance with

This paper is drawn from the research supported by the IT R\&D program of MIC (Ministry of Information and Communication Republic of Korea) and IITA (Institute for Information Technology Advancement) [2005-S-404-33, 3G Evolution Mobile Terminal Technology]. lower cost, a user might wish to connect to the WLAN access network as long as possible.

In 3G-WLAN overlay network, when a UE (User Equipment) currently connecting to $3 \mathrm{G}$ network enters to WLAN coverage, it can determine on changing the connection into WLAN. If the UE decides and executes the handover to WLAN, it has to carry out the series of procedures like WLAN access procedures including an AP (Access Point) association, a subscriber authentication and an IP address allocation, and also like the IP layer mobility operations $[7,8,9,10]$. In this case, as the UE can break off the $3 \mathrm{G}$ link after completing of these basic procedures. Accordingly, the link break time is insignificant and there is no impediment in a service.

But in case of the handover to $3 \mathrm{G}$ network from WLAN, the UE is apt to lose the WLAN signal without any notice by moving and it can be apart from the WLAN coverage suddenly before connecting to $3 \mathrm{G}$ network. Hence the services are disrupted by unexpected link corruption and the handover latency becomes very serious problem. Accordingly in this case, the most important thing the UE has to do is to execute the handover to $3 \mathrm{G}$ before losing the WLAN signal in link layer as soon as possible. However, until the service is connected to $3 \mathrm{G}$ network, it is requested that the many signaling procedures for $3 \mathrm{G}$ access and additionally IP mobility operations. The $3 \mathrm{G}$ access procedures include the service request procedure with subscriber authentication and the PDP (Packet Data Protocol) context activation procedure with IP address allocation. If it takes too much time to finish those, handover latencies are increased and in conclusion the service gets to fail.

If the link layer can previously predict the lost of WLAN signal and the connection to $3 \mathrm{G}$ network can be made in advance before link disruption, the link break time is quite decreased and the services can be continued without any interruption. Hence the handover prediction for link triggering is very important for seamless mobility.

In this paper, we consider the five handover prediction algorithms for applying to the handover to $3 \mathrm{G}$ network from WLAN. The handover prediction algorithms previously decide the handover based on various data which are periodically gathered by the UE such as RSS (Received 
Signal Strength), velocity, direction, and history. We compare them with correct prediction ratios by simulation.

The rest of this paper is organized as follows: In section II, we address the $3 \mathrm{G}$ and WLAN overlay network structure and describe the five handover prediction algorithms. And the simulation results are shown in section III. Finally, we conclude the paper with remarks.

\section{HANDOVER PREDICTION}

Generally, $3 \mathrm{G}$ operators want to provide WLAN services in hot-spots and so it may be easily assumed that WLAN systems are deployed in the same operator's $3 \mathrm{G}$ coverage with overlapped structure.

Fig. 1 shows an example of overlay network model for supporting 3G-WLAN interworking.

For minimizing the handover latency when the UE gets away from WLAN coverage, we consider the five prediction algorithms which have the input data as RSS with threshold, movement extrapolation, history data, mobility pattern, and distance between a UE and an AP.

\section{A. Prediction Algorithm based on RSS with threshold}

The RSS based handover prediction algorithm is well known mechanism [6, 11]. Generally, a UE periodically measures the RSS from the current WLAN AP and compares it with a predefined threshold, e.g., $-80 \mathrm{dBm}$. If the RSS is below the threshold, it searches neighboring APs that have the stronger RSS than current one. If there is a serving AP with strong RSS, the UE initiates the handovers to the new AP, which may be based on the IAPP (Inter Access Point Protocol) of IEEE 802.11f [12]. However, if there is no proper AP, it initiates the handover to $3 \mathrm{G}$ as shown in fig. 2 . This is very simple and fast, but it well acts in small coverage and is suitable for only horizontal handover. Also as the RSS is apt to frequently change according to the radio characteristic like fading and path loss, this scheme undergoes performance deterioration in the area such as a downtown with many obstacles and induces much ping-pong problems.

\section{B. Prediction Algorithm based on Movement Extrapolation}

This algorithm predicts the handover based on the movement of a UE $[11,13,14]$. It extrapolates the UE's movement using its current location, direction, and velocity and expects the next location $\mathrm{L}[\mathrm{x}, \mathrm{y}]$ at distance $\mathrm{D}$ from the current location after certain periods as shown in fig. 3. It finds a serving AP of the location $\mathrm{L}$ and then if it is different from the current AP, it initiates the handover to the AP. If it fails to find a proper serving AP, it initiates the handover to $3 \mathrm{G}$ network.

As it precisely predicts handover based on its location information, the performance never falls down regardless of any obstacles. If the movement of the UE is uniform motion of straight line, moreover, this algorithm can achieve a perfect handover prediction.

\section{Prediction Algorithm based on Handover History Data}

This algorithm predicts the handover based on history data which records the passed paths of a UE $[6,13]$. When the UE is moving, it gathers the cell information about the current cell and previous cell and stores it as the history data as shown in fig. 4.

The history data include the information for each AP and $3 \mathrm{G}$ UTRAN and provide the number of handover that occurred between APs or from AP to 3G UTRAN. The UE selects an AP to which the most many handover was happened. If there is no proper AP, it initiates a handover to $3 \mathrm{G}$ network.

The stored data are classified according to the number of handover that the UE achieves before. The UE predicts the next cell for handover according to the history.

This algorithm is applied to a variation of UE's movement. Although this algorithm has to make and maintain many history data, it can predict the handover more precisely.

\section{Prediction Algorithm based on Mobility Pattern}

This algorithm predicts a handover by considering mobility pattern of a UE $[11,13]$. It also gathers the history data about the UE's movement and extracts the mobility patterns based on the history data. It shows in fig. 5.

The mobility patterns are used as input data for predicting the next served AP or UTRAN. By comparing the current mobility pattern of the UE with the old mobility patterns, the link layer predicts the handover and triggers the execution of it.

As this algorithm reflects more movement variations than the simple history based algorithm, it predicts the handover more precisely even though the UEs maintain more mobility patterns according to its movement. Also, there is another mobility prediction technique by real-time mobile positioning and road topology information [15].

\section{E. Prediction Algorithm based on Distance from AP}

This algorithm predicts a handover based on the current position of UE which is apart from the present associated AP. $[6,11]$. The UE compares the distance from the current associated AP with the distance from near APs of neighbor cells as shown in fig. 6 .

When a UE is getting away from the current AP, it calculates the time when it will get out of. If it determines that it will be out in several times later, it decides the handover and searches the next nearest AP. If there is nearer AP of some neighbor cells than the current associated AP, the UE determines the handover to the nearest AP. If there is no any one, it triggers the handover to $3 \mathrm{G}$ network.

As this algorithm refers only the location information of the UE which is apart from some AP of the cells, it can be apt to determine the handover faster than others but less precise because it doesn't consider any information of the UE's movement or velocity. 


\section{PERFormance ANAlysis}

We have simulated the above five prediction algorithms to analyze the performance in WLAN and $3 \mathrm{G}$ overlay network environments.

\section{A. Network Model}

Fig. 7 shows a network model for our simulation, which consists of several WLANs dispersed in hot-spots of $3 \mathrm{G}$ networks.

The UE has two types of mobility models: a random waypoint mobility model and a realistic road mobility model. In the random waypoint model, initially each UE is at a random position within the simulation area. The UE randomly chooses a new location and moves to the chosen location in each several milliseconds. Its velocity is constant and the speed is randomly chosen between A meters/sec and B meters/sec.

In realistic road mobility model alternatively, a UE is positioned at one of the roads. In every several milliseconds, a UE chooses a location on one of the roads to be its next destination. The UE will traverse towards this destination on one of the shortest paths calculated using Dijkstra's shortest path algorithm. The UE will move at a pre-defined speed towards this destination. Once the destination is reached, the UE will again choose another location on any one of these roads and repeat this movement.

In the overlapped network environment, a UE executes several handovers in variable positions as shown in fig. 7. It executes horizontal handover in point (a) and (d) and executes vertical handover in point $\square$ and $\square$.

As our target is for evaluating the performance of prediction algorithms in vertical handover, we collect and analyze the results in only cases that the UE executes the vertical handover like as the point and .

We implemented the algorithms using Visual $\mathrm{C}++$ and simulated them on the Qualnet 3.8 platform on Windows XP.

\section{B. Results and Analysis}

Fig. 8 shows the correct prediction ratios of the five handover prediction algorithms for the realistic road mobility model with 10 UEs. As displayed in the figure, the mobility pattern based algorithm shows the best performance.

The history data based algorithm shows similar graph with the mobility pattern based algorithm. However, this has lower accuracy because it reflects less user mobility characteristic.

The mobility patter based algorithms and the history data based algorithms suffer from burdens which should maintain massive data compared with other three algorithms. The movement extrapolation based algorithm predicts a handover according to the direction and the speed of a node. If the node suddenly doesn't change its direction, the handover generally occurs according to the direction of the node and accordingly its accuracy is better than the other two algorithms RSS based and distance based algorithms. As these two algorithms don't consider the mobility characteristic, the accuracies of them are very low.

Fig. 9 shows the correct prediction ratios of the five handover prediction algorithms for random waypoint mobility model for 10 UEs. It looks similar to fig. 8 but the accuracy of the mobility pattern based algorithm is much decreased and also that of the history data based algorithm is slightly declined. But the movement extrapolation based algorithm shows much better performance than realistic road mobility model. The other algorithms are not so affected.

Each algorithm operates independently and does not affect each other. In conclusion, the algorithms based on the history data and the mobility pattern show higher accuracies in realistic road mobility model and the movement extrapolation based algorithm shows the more accurate result in random waypoint mobility model.

\section{CONCLUSION}

The integration of the $3 \mathrm{G}$ and WLAN has been identified as an important feature for future wireless systems. Seamless mobility is a final goal of 3G-WLAN interworking system, which seems to be hard to achieve. For achieving the seamless mobility, it is needed to reduce the handover latency first of all.

When a UE is moving away from WLAN coverage, it is apt to lose the WLAN signal without any notice and can be apart from the WLAN coverage suddenly before connecting to 3G. In this case the services are disrupted by unexpected link corruption. Hence, handover prediction algorithm is very important issue for guaranteeing the seamless mobility.

If the link layer previously detects the link corruption and triggers the handover beforehand, the UE can execute the signaling operations for $3 \mathrm{G}$ access before breaking off the WLAN connection and so the services can be continuous without any interruption.

This paper showed our application of the several handover prediction algorithms for minimizing the handover latency, especially in case of the handover to $3 \mathrm{G}$ network from WLAN. The handover prediction algorithms previously decide the handover to a new AP or $3 \mathrm{G}$ network using various data periodically gathered by the UE. They are based on RSS, velocity, direction, history data, mobility pattern, and distance. We also showed the comparison results between them with correct prediction ratios by simulation.

These algorithms had been measured in different mobility environments, random waypoint mobility model and realistic road mobility model. In the result of the simulation, the mobility pattern based algorithm shows remarkable performance in realistic road mobility model and the movement extrapolation based algorithm shows the more accurate prediction result in random waypoint mobility model. For minimizing the handover latency by fast link layer triggering, we can use these prediction algorithms in each acceptable environment. 


\section{REFERENCES}

[1] 3GPP TS 23.934; "3GPP system to Wireless Local Area Network (WLAN) Interworking; Functional and architectural definition (Release 6)," 3GPP TSG SA, Aug. 2002.

[2] 3GPP TS 23.234, "3GPP system to Wireless Local Area Network (WLAN) interworking; System description (Release 6)," 3GPP TSG SA, March 2005

[3] Bernaschi, F. Cacace, and G. Iannello, “ Vertical Handoff Performance in Heterogeneous Networks," Proc. of the Int. Conf. on Parallel Processing Workshops (ICPPW'04), 2004

[4] K. Pahlavan et al., "Handoff in Hybrid Mobile Data Networks" IEEE Pers. Commun., April. 2000.

[5] M. Buddhikot, G. Chandranmenon, S. Hand, Y.W. Lee, S. Miller and L. Salgrelli, "Integration of 802.11 and third generation wireless data networks," Proc. of INFOCOM, 2003, pp.503-512.

[6] M. Lott, M. Siebert, S. Bonjour, D. von Hugo and M. Weckerle, "Interworking of WLAN and 3G Systems," Proc. Of IEE Commune. Vol. 151, No. 5, October 2004

[7] D. Johnson, C. Perkins, and J. Arkko, "Mobility Support in IPv6," IETF RFC 3775, June 2004.
[8] IETF RFC 4140, H. Soliman, C. Catelluccia, K. E. Malki, and L. Bellier, Hierarchical Mobile IPv6 Mobility Management, Aug. 2005.

[9] IETF RFC 4068, R. Koodli, Fast Handovers for Mobile IPv6, July 2005.

[10]H. Y. Jung, E. A. Kim, J. W. YI, and H. H. Lee, "A Scheme for Supporting Fast Handover in Hierarchical Mobile IPv6 Networks," ETRI Journal, vol.27, no.6, Dec. 2005, pp.798-801.

[11] G. P. Pollini, “Trends in handover design,” IEEE Commu. Magazine, March 1996.

[12]IEEE, "Recommended Practice for Multi-Vendor Access Point Interoperability via an Inter-Access Point Protocol Across Distribution Systems Supporting IEEE 802.11 Operation,” IEEE Std 802.11f/D5, Jan. 2003.

[13]J. Chan, A. Seneviratne. "A practical user mobility prediction algorithm for supporting adaptive QoS in wireless networks," Proc. of IEEE Int. Conf. on Networks (ICON '99), Sep. 1999, pp. 104-111.

[14]A. Jayasuriya and J. Asenstorfer, "Mobility Prediction Model for Cellular Networks based on the Observed Traffic Patterns," Proc. Of Wireless and Optical Communications (WOC), 2002.

[15]W. Soh, and H. S. Kim, "QoS provisioning in cellular networks based on mobility prediction techniques," IEEE Commune. Magazine, 2003, pp. $86-92$

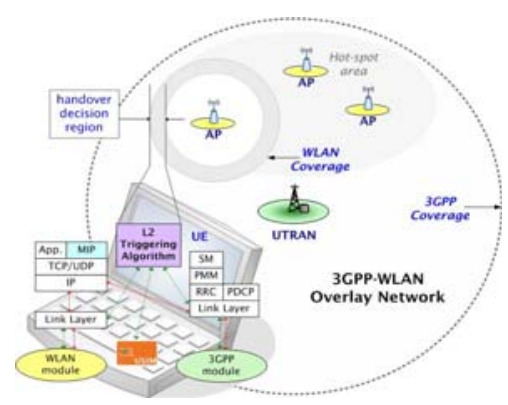

Figure 1. Overlay Network Model

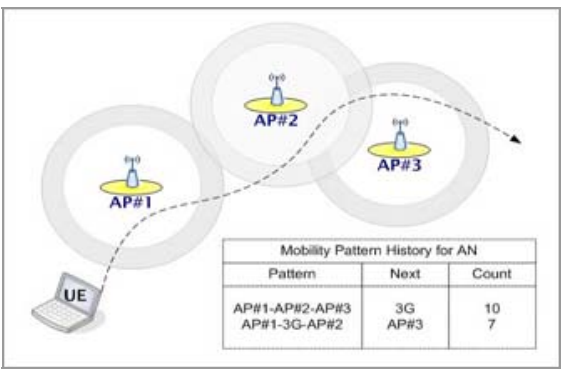

Figure 4. Mobility Pattern based Prediction

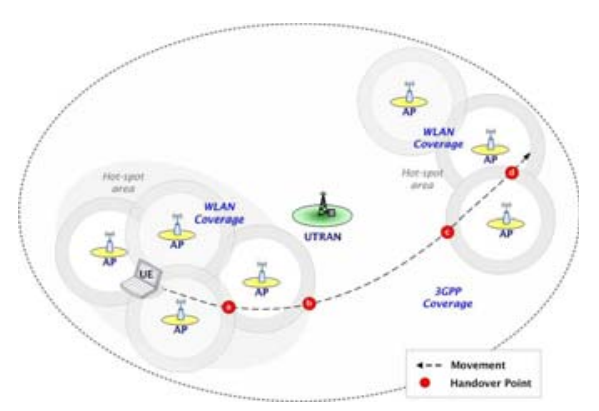

Figure 7. Simulation model

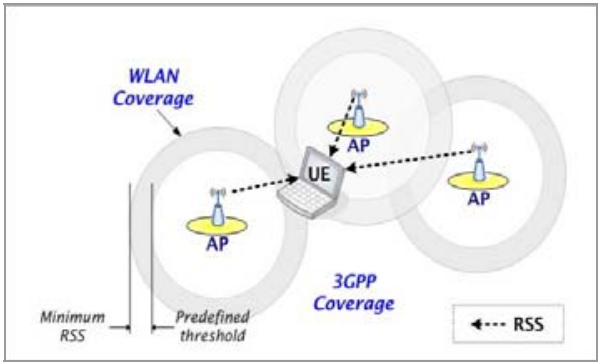

Figure 2. RSS based prediction

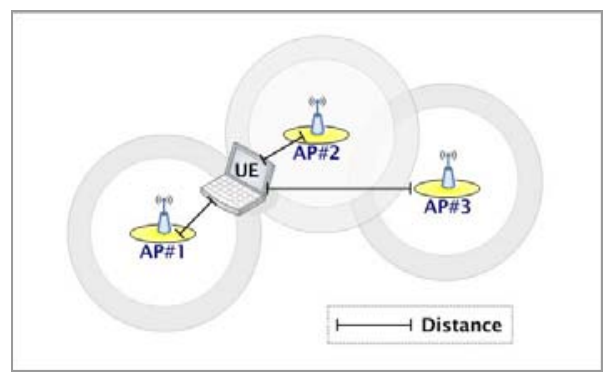

Figure 5. distance based Prediction

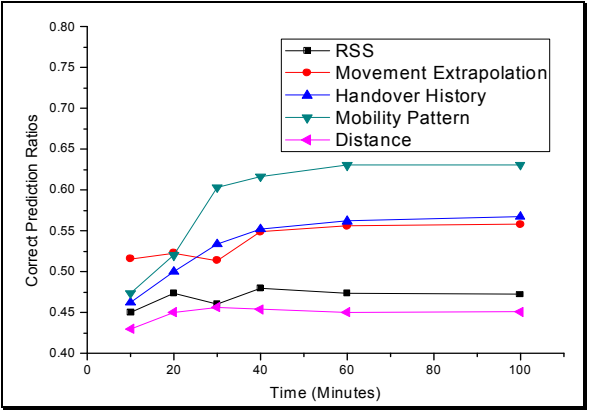

Figure 8. Correct prediction ratios for realistic road mobility model

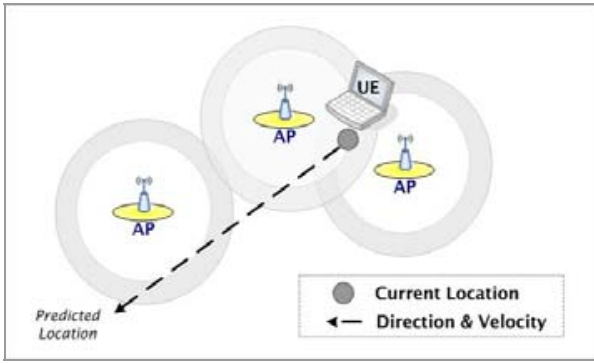

Figure 3. Movement Extrapolation based prediction

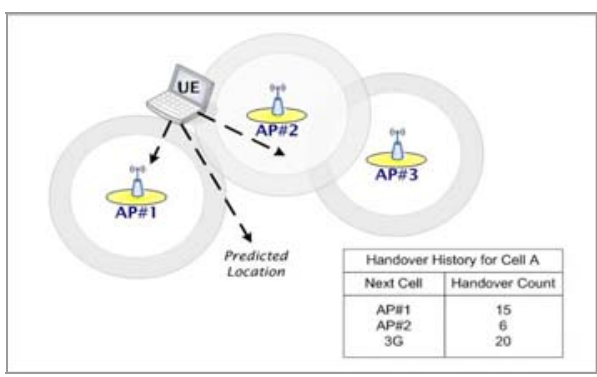

Figure 6. History Data based prediction

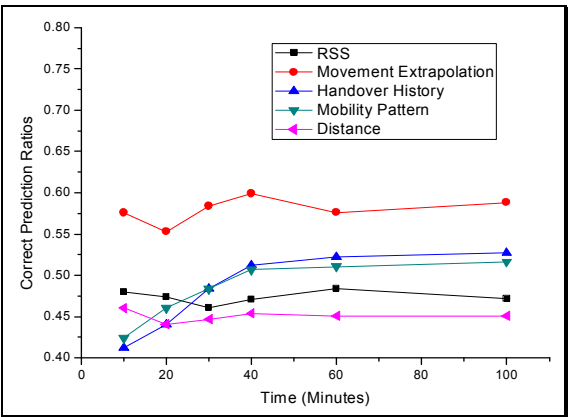

Figure 9. Correct prediction ratios for random waypoint mobility model 